\title{
Treatment beyond progression with chemo-immunotherapy in an advanced esophageal squamous cell carcinoma patient: a case report
}

\author{
Ping Peng ${ }^{1 \#}$, Yajie Xiao ${ }^{2 \#}$, Zhikun Zhao ${ }^{2}$, Chao Sun ${ }^{2}$, Dongfang $\mathrm{Wu}^{2}$, Yuan Chen ${ }^{1}$, Li Zhang $^{1}$ \\ ${ }^{1}$ Department of Oncology, Tongji Hospital, Tongji Medical College, Huazhong University of Science and Technology, Wuhan, China; ${ }^{2}$ YuceBio \\ Technology Co., Ltd., Shenzhen, China \\ \#These authors contributed equally to this work. \\ Correspondence to: Yuan Chen; Li Zhang. Department of Oncology, Tongji Hospital, Tongji Medical College, Huazhong University of Science and \\ Technology, Jie Fang Road No. 1095, Wuhan, China. Email: chenyuan008@163.com; luzigang@163.com.
}

\begin{abstract}
Esophageal cancer is an aggressive and common malignancy in Asian countries. Due to late diagnosis and limited treatments, the prognosis of esophageal cancer is still very poor. Although immune checkpoint inhibitors have become promising second-line treatments for esophageal cancer, there are limited evidences for first-line treatments. Here, we reported a case of successful treatment beyond progression with chemo-immunotherapy for esophageal squamous cell carcinoma (ESCC). Combined with local resection of several metastases during chemo-immunotherapy, the patient achieved a long survival time of 22 months and a good quality of life. Samples of the primary tumor and three metastases of testicle, skin nodule and left adrenal were obtained to perform whole exome sequencing (WES), RNA sequencing and immunohistochemistry. The skin nodule metastasis was resected after partial response, while the other two metastases of testicle and adrenal gland were removed after disease progression. Immunohistochemistry results exhibited low/negative PD-L1 expression and WES results showed intermediate TMB and MSI-L for all three lesions. However, RNA sequencing results presented a higher percentage of infiltrating CD8 ${ }^{+}$ $\mathrm{T}$ cells, higher signature scores of $\mathrm{T}$ cell status and higher expression level of human leukocyte antigen (HLA) genes in skin nodule metastasis than the other two metastases. This case provided a clinical evidence of beneficial treatment beyond progression with chemo-immunotherapy for ESCC. In addition, tumor microenvironment might be essential for clinical responses at the sampling time point.
\end{abstract}

Keywords: Esophageal squamous cell carcinoma (ESCC); chemo-immunotherapy; treatment beyond progression; tumor microenvironment; case report

Submitted Jul 23, 2021. Accepted for publication Oct 15, 2021.

doi: $10.21037 /$ tcr-21-1395

View this article at: https://dx.doi.org/10.21037/tcr-21-1395

\section{Introduction}

Esophageal cancer ranks the top cancers with high mortality globally, and above half of new patients are diagnosed in China annually (1), among which esophageal squamous cell carcinoma (ESCC) accounts for about $90 \%$ of esophageal cancer cases (2). Surgery is the most important treatment for esophageal cancer, but most patients with esophageal cancer have been diagnosed at an advanced stage and have lost the opportunity for surgery. Consequently, lacking of early-stage diagnosis and limited treatment, the prognosis of esophageal cancer is still poor.

To date, programmed cell death 1 (PD-1) inhibitors have revolutionized the treatment of solid tumors (3), especially in multi-line treatments for advanced esophageal carcinoma (4-7). Recently, FDA approved pembrolizumab for second-line treatment of $\mathrm{PD}-\mathrm{L} 1$ positive recurrent locally advanced or metastatic ESCC based on the results of KEYNOTE-180 study (7) and KEYNOTE-181 study (5). 

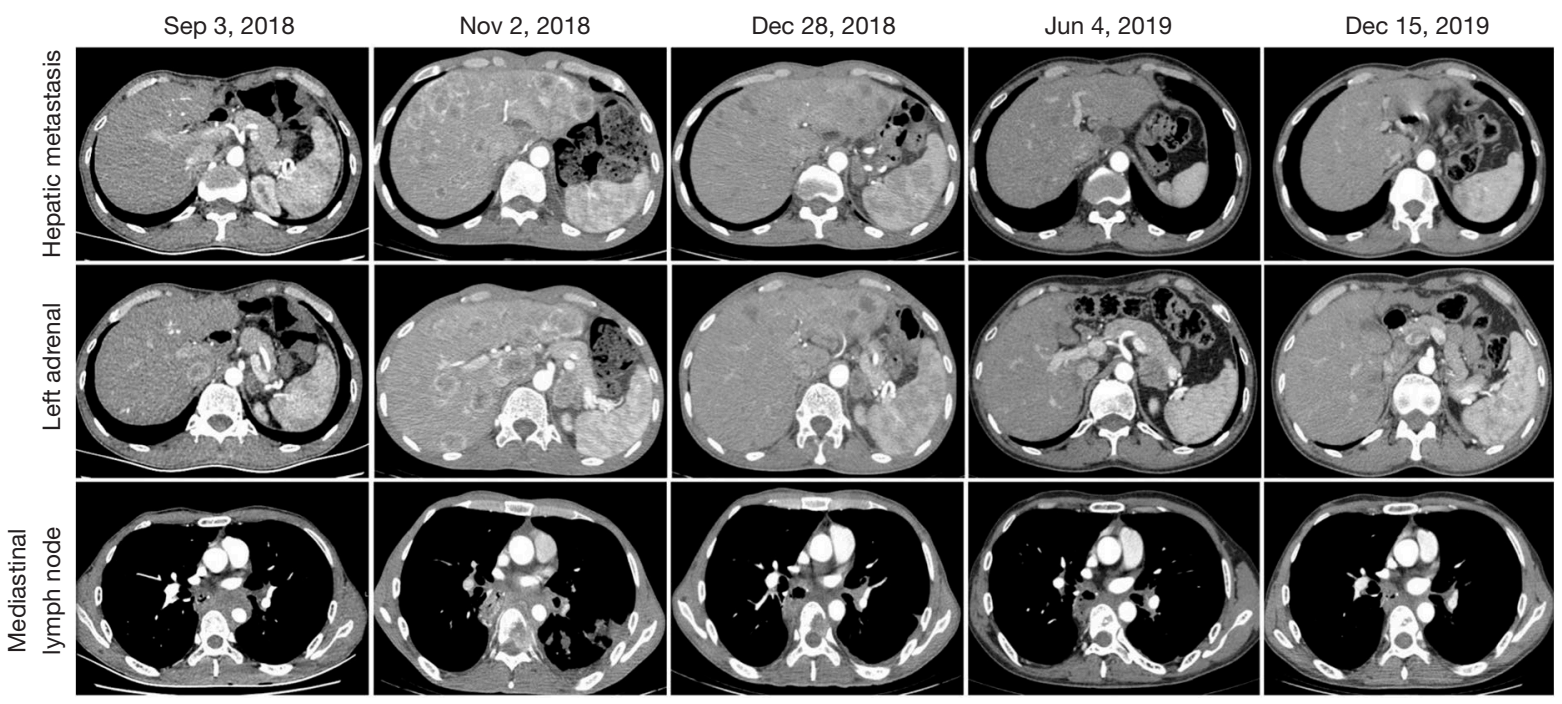

Figure 1 CT images of liver metastasis, the left adrenal metastasis and mediastinal lymph node metastasis during treatment.

Notably, most of ESCC patients received monoimmunotherapy or posterior line treatments. However, there are very few published results of front-line chemoimmunotherapy for ESCC patients.

Here, we reported an ESCC patient receiving successful treatment beyond progression (TBP) with front-line chemo-immunotherapy. Overall, this patient achieved a long survival time of 22 months and a good quality of life. Clonal evolution process and tumor microenvironments were also analyzed to reveal the potential mechanism for chemo-immunotherapy in this case. We present the following case in accordance with the CARE reporting checklist (available at https://dx.doi.org/10.21037/tcr-211395).

\section{Case presentation}

\section{Clinical diagnosis, treatments, and outcomes}

A 56-year-old man received radical surgery for esophageal carcinoma in August 23th, 2017, and was diagnosed as pathologic stage IIB ESCC (PT2N1M0). After the surgery, no adjuvant therapy was given and the patient was followed up for every three months. Nearly a year later, CT scan showed multiple metastatic lesions in the liver, left adrenal and mediastinal lymph node on September 3rd, 2018 (Figure 1). Besides, multiple nodules of approximately $1.5 \mathrm{~cm}$ in diameter was observed subcutaneously on the right shoulder of the patient. Subsequently, the patient underwent a fine needle aspiration of subcutaneous nodule and the cytopathology result confirmed metastatic squamous-cell carcinoma. Then, he received first-line chemo-immunotherapy with pembrolizumab $(2 \mathrm{mg} / \mathrm{kg}$, Q3W) and docetaxel plus cisplatin (docetaxel $75 \mathrm{mg} / \mathrm{m}^{2}$; cisplatin $75 \mathrm{mg} / \mathrm{m}^{2}$, Q3W) for 2 cycles from Sep 8th, 2018 (Figure 2). Unfortunately, the physical examination one month later revealed a new solid lesion in right testicle, which was proved to be squamous-cell carcinoma after a testectomy. At that time, subcutaneous nodules were unchanged. CT scan on Nov 2th, 2018 showed enlarged metastases in the liver and left adrenal (Figure 1). According to RECIST 1.1 criteria, the patient achieved a progressive disease (PD).

Recently, some patients with solid tumors have been demonstrated to still benefit from immunotherapy beyond progression. Given that PD-1 inhibitors were given for just 2 cycles and no other treatment-related side effects happened, the patient was continued with the same chemoimmunotherapy regimen for another 2 cycles from Nov 5 th, 2018. Interestingly, the volume of the subcutaneous nodules on the right shoulder reduced to half in diameter. Based on CT scan on Dec 28th, 2018, all lesions in the mediastinum, liver and left adrenal shrunk and the patient obtained a partial response (PR) (Figure 1).

Then, the patient was treated with pembrolizumab monotherapy as maintenance treatment $(2 \mathrm{mg} / \mathrm{kg}, \mathrm{Q} 3 \mathrm{~W})$ for 7 cycles from Dec 30th, 2018. Despite no changes in lymph nodes in mediastinum and hepatic metastasis stayed the same, an enlarged left adrenal metastasis was found in CT scans on June 4th, 2019 (Figure 2). Considering only the 


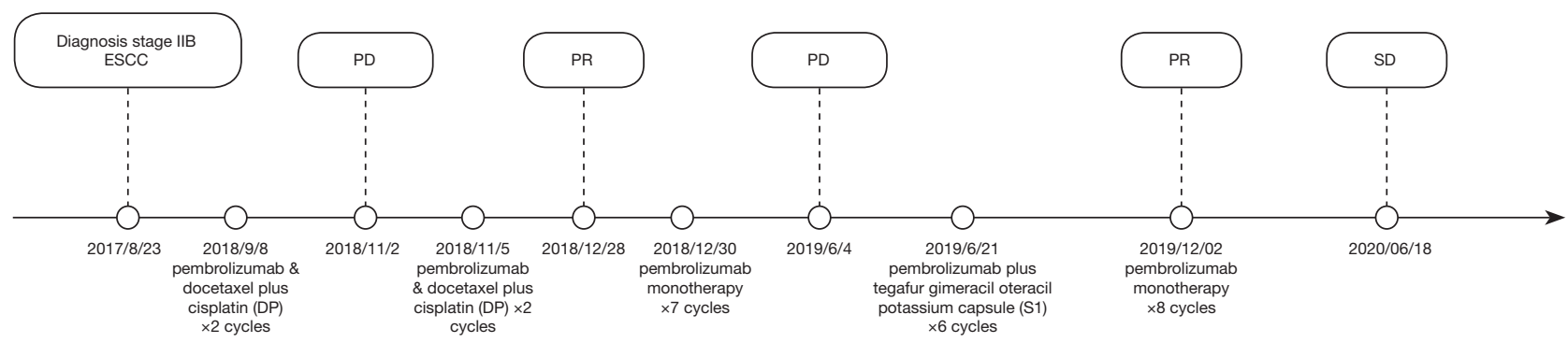

Figure 2 Treatment courses and clinical outcomes of this patient. ESCC, esophageal squamous cell carcinoma; PD, progressive disease; SD, stable disease; PR, partial response.

disease progression in left adrenal, the patient underwent left adrenal lumpectomy and pathological result showed squamous-cell carcinoma. Therefore, the patient was switched to the second line treatment with pembrolizumab (2 $\mathrm{mg} / \mathrm{kg}$, Q3W) plus tegafur gimeracil oteracil potassium capsule (S1) chemotherapy (60 mg bid, D1-14, Q3W) from June 21th to Nov 1st, 2019 to for 6 cycles, and reached a stable disease (SD) with similar size of metastatic lesions in mediastinum lymph nodes and liver (Figure 2). Then, the patient continued to receive pembrolizumab monotherapy ( $2 \mathrm{mg} / \mathrm{kg}, \mathrm{Q} 3 \mathrm{~W}$ ) as maintenance therapy for 8 cycles until June 20th, 2020, and retained SD as before. He was tolerant without any adverse effects and willing to continue with current therapeutic regimens.

\section{PD-L1 staining, whole exome sequencing (WES) and $R N A$ sequencing ( $R N A-$ seq) results}

To better understand the tumor progression process and tumor environments, the tumor tissues of resected primary tumor on Aug 23, 2017 (TE), testicle on Oct 22, 2018 (TT), skin nodule on Dec 12, 2018 (TS), and left adrenal on Jun 13, 2019 (TA) were collected to perform PD-L1 staining, WES and RNA-seq. The combined positive scores (CPS) of PD-L1 expression in four tumor tissues (TE, TT, TS, TA) were $0,0,0$ and 3, respectively (Figure 1). A total of 60 non-synonymous mutations were identified in all four samples (Figure $3 A$ ), and averagely accounted for $41 \%$ of non-synonymous mutations in each sample (Figure $3 B$ ). Except TE, about 38\% mutations in all three metastases as well as mutational signatures were similar (Figure 3C). These results demonstrated the same origin of all four tumors. Therefore, the potential evolutional process was conducted based on the estimated cancer cell fractions of all mutations (Figure 3D). Each metastasis was derived from a subclone of another tissue, accompanied by newly acquired clonal and sub-clonal mutations. In addition, the tumor mutational burden (TMB) levels were intermediate, ranging from 4.0 to $5.2 \mathrm{mut} / \mathrm{Mb}$ (Figure $3 E$ ). No mismatch repair (MMR) related mutations, immune-resistant or hyperprogression related mutations or loss of heterogeneity at human leukocyte antigen $(H L A)$ genes were detected. All four samples were affirmed as microsatellite instability (MSI)-L. Significantly, the RNA-seq results showed the highest proportions of $\mathrm{CD} 8^{+} \mathrm{T}$ cells and macrophage $\mathrm{M} 1$ (Figure $3 F$ ), highest signature scores of $\mathrm{T}$ cell cytotoxicity, $\mathrm{T}$ cell co-stimulatory and $\mathrm{T}$ cell exhaustion (Figure $3 G$ ), higher $H L A$ genes expression in TS (Figure $3 E$ ). But, the proportion of $\mathrm{CD}^{+} \mathrm{T}$ cell was the lowest in TS. These indicated a hot microenvironment in TS, which might contribute to better response at that sampling time.

All procedures performed in studies involving human participants were in accordance with the ethical standards of the institutional and/or national research committee(s) and with the Helsinki Declaration (as revised in 2013). Written informed consent was obtained from the patient for publication of this case report and accompanying images. A copy of the written consent is available for review by the editorial office of this journal.

\section{Discussion and conclusions}

Here, we reported beneficial TBP with front-line chemoimmunotherapy in an advanced ESCC patient. Four tissue samples from different organs were biopsied throughout the treatment course, and comprehensive bioinformatics analyses were performed to clarify potential molecular mechanisms.

Some clinical studies have already demonstrated promising activity and manageable safety of PD-1 inhibitors in ESCC patients (5,7-10). For example, the stage Ib KEYNOTE-028 trial showed an ORR rate 
A
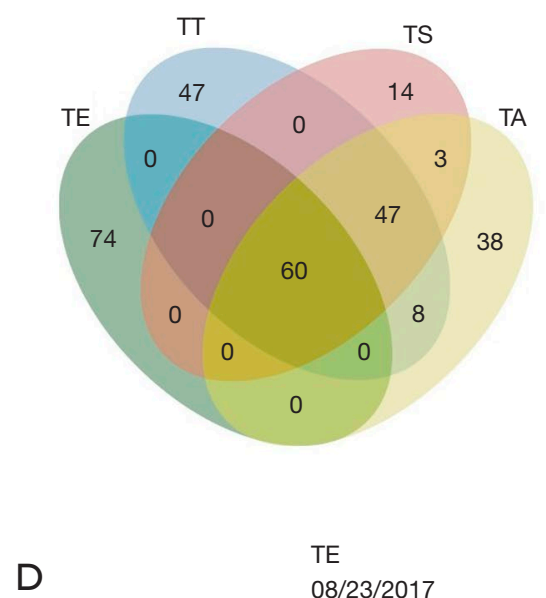

TE $08 / 23 / 2017$

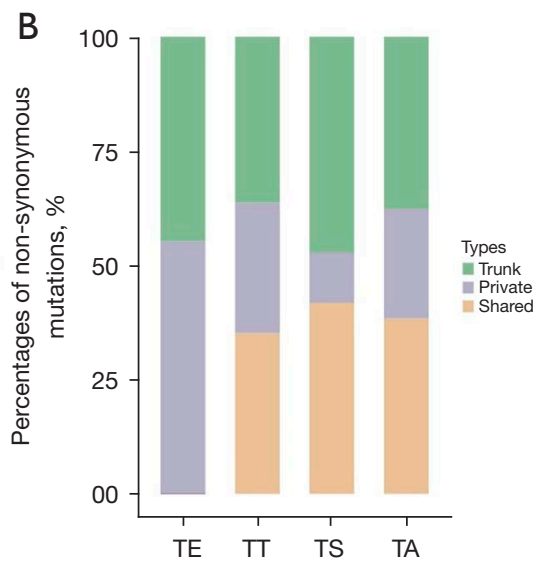

$\pi$ $10 / 22 / 2018$

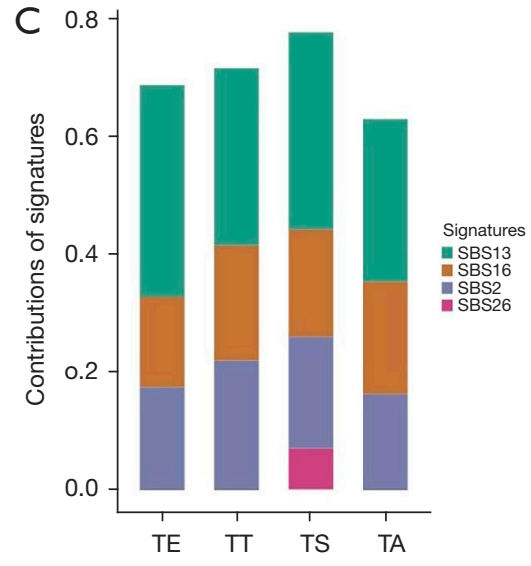

TA $06 / 13 / 2019$

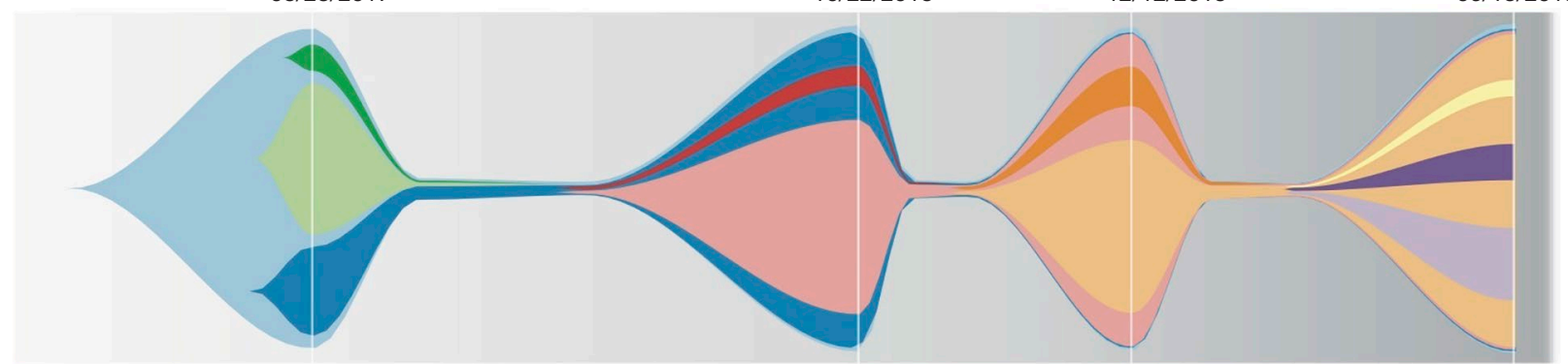

E

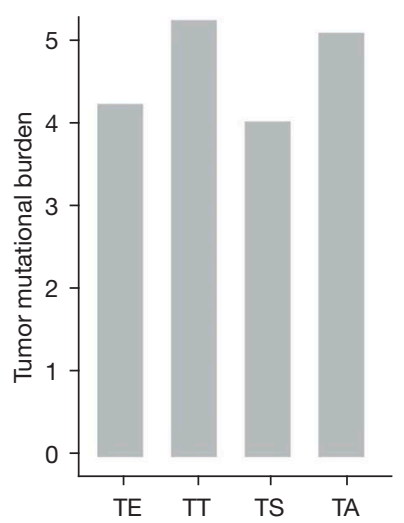

$\mathrm{F}$

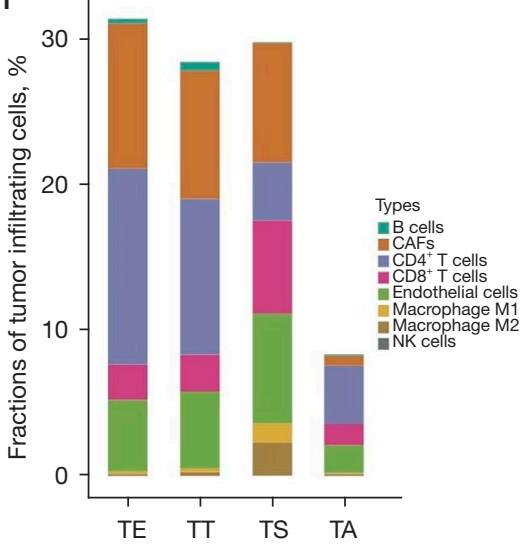

G

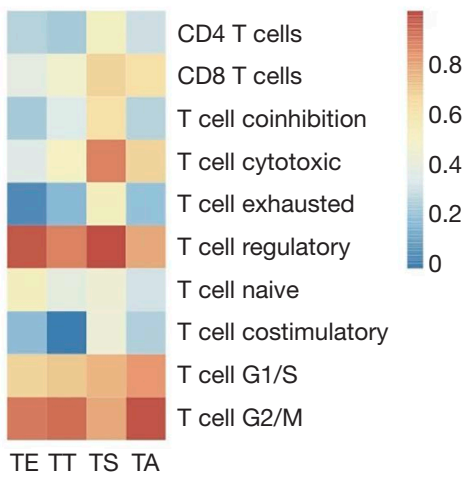

Figure 3 Genomic and transcriptomic features of all samples. (A) Venn diagram of all detected non-synonymous mutations; (B) percentages of non-synonymous mutations; (C) contributions of mutational signatures; (D) schematic diagram of evolutional process of the four samples. Each color denotes a group of tumor cells; (E) HLA gene expression; (F) estimated fractions of tumor infiltrating cells; (G) T cell related expression signature scores. TE, primary tumor; TT, testicle tissue; TS, skin nodule tissue; TA, left adrenal tissue; Trunk, mutations detected in all four samples; Shared, mutations shared by two or three samples; Private, mutations detected in only one sample; SBS, single base substitution; CAFs, cancer associated fibroblasts; NK cells, natural killer cells.

of $30 \%$ (95\% CI: $13 \%$ to $53 \%$ ) with no grade $4-5$ adverse events in patients PD-L1-positive advanced esophageal tumors treating with pembrolizumab monotherapy (9). In phase II KEYNOTE-180 study, an ORR rate of $14.3 \%$
(95\% CI: $6.7 \%$ to $25.4 \%$ ) was observed in ESCC patients receiving pembrolizumab treatment (7). Furthermore, KEYNOTE-181 study revealed that pembrolizumab can prolong OS (8.2 months) versus chemotherapy 
(7.1 months) as second-line treatment for advanced esophageal cancer patients (HR 0.78; 95\% CI: 0.63-0.96; $\mathrm{P}<0.01$ ) (5). However, studies focusing on first-line therapy with immunotherapy alone or in combination with chemotherapy in ESCC patients are still ongoing. Recently, improved OS was observed in advanced oesophageal squamous cell carcinoma patients following first-line therapy with pembrolizumab plus chemotherapy (12.6 months) versus chemotherapy alone (9.8 months) (HR 0.72; $95 \% \mathrm{CI}$ : $0.60-0.88 ; \mathrm{P}<0.0001)(10)$.

Interestingly, patients receiving immunotherapy can still benefit from TBP in melanoma, non-small cell lung cancer (NSCLC) and clear-cell renal carcinoma (4,6,11-13). At times, some NSCLC patients also received local treatment for progressive lesions during immunotherapy (13). Therefore, more treatment courses and multiple evaluations are needed to confirm the efficacy. In this case, the patient did not achieve PR until twice evaluation after 4 cycle chemo-immunotherapy. He felt discomfort temporarily when disease progressed and recovered quickly after local surgery. Overall, this patient achieved a long survival time of 22 months and a good quality of life. This case may indicate that ESCC patients might also benefit from TBP during chemo-immunotherapy accompanying by local resection of metastases.

Additionally, further analyses were performed for all four tumor samples which were collected thought the therapy to reveal the potential molecular mechanisms. All four samples showed intermediate TMB levels and MSI-L status, low/negative PD-L1 expression together with no typical immunological features. Despite of similar genetic features, TS was observed with highest proportion of $\mathrm{CD}^{+} \mathrm{T}$ cells and macrophage M1, highest signature score of $\mathrm{T}$ cell cytotoxicity, $\mathrm{T}$ cell co-stimulatory and $\mathrm{T}$ cell exhaustion, lowest proportion of $\mathrm{CD}^{+} \mathrm{T}$ cell. Likewise, levels of infiltrated $\mathrm{CD}^{+} \mathrm{T}$ cells can be increased during therapy in responding tumors (14). Moreover, high density of infiltrated $\mathrm{CD}^{+} \mathrm{T}$ cells was observed to be associated with improved clinical outcomes in NSCLC patients (15). Therefore, tumor microenvironments in TS might be important for PR during the whole therapy. On the other side, highest expression score of HLA genes were observed in TS, consistent with the infiltrating levels of $\mathrm{CD}^{+} \mathrm{T}$ cells. It was proved that downregulation of $H L A$ genes can reduce antigen presentation and prevent tumor recognition by $\mathrm{CD}^{+} \mathrm{T}$ cells, which may facilitate immune evasion and tumor progression $(16,17)$ in turn. So, the lower expression of $H L A$ genes in other two metastases might contribute to the tumor progression and poor response to chemoimmunotherapy.

In conclusion, this case presented successful treatment beyond progression with chemo-immunotherapy for advanced esophageal cancer. In addition, tumor microenvironment might play a key role for clinical responses at the sampling time point.

\section{Acknowledgments}

The authors thank the patient and patient's kin for agreement to publication of the report.

Funding: This study was funded by the Wu Jieping Medical Foundation (320.6750.19078), Chinese Society of Clinical Oncology Foundation (Y-BMS2019-070) and Hubei Provincial Natural Science Foundation of China (grant No. 2019CFB544).

\section{Footnote}

Reporting Checklist: The authors have completed the CARE reporting checklist. Available at https://dx.doi. org/10.21037/tcr-21-1395

Conflicts of Interest: All authors have completed the ICMJE uniform disclosure form (available at https://dx.doi. org/10.21037/tcr-21-1395). PP, YC, and LZ report that since the initial planning of the work, this study was funded by the Wu Jieping Medical Foundation (320.6750.19078), Chinese Society of Clinical Oncology Foundation (Y-BMS2019-070) and Hubei Provincial Natural Science Foundation of China (grant No. 2019CFB544). No other funding or supports were obtained for the past 36 months. The other authors have no conflicts of interest to declare.

Ethics Statement: The authors are accountable for all aspects of the work in ensuring that questions related to the accuracy or integrity of any part of the work are appropriately investigated and resolved. All procedures performed in studies involving human participants were in accordance with the ethical standards of the institutional and/or national research committee(s) and with the Helsinki Declaration (as revised in 2013). Written informed consent was obtained from the patient for publication of this case report and accompanying images. A copy of the written consent is available for review by the editorial office of this journal.

Open Access Statement: This is an Open Access article 
distributed in accordance with the Creative Commons Attribution-NonCommercial-NoDerivs 4.0 International License (CC BY-NC-ND 4.0), which permits the noncommercial replication and distribution of the article with the strict proviso that no changes or edits are made and the original work is properly cited (including links to both the formal publication through the relevant DOI and the license). See: https://creativecommons.org/licenses/by-nc-nd/4.0/.

\section{References}

1. Bray F, Ferlay J, Soerjomataram I, et al. Global cancer statistics 2018: GLOBOCAN estimates of incidence and mortality worldwide for 36 cancers in 185 countries. CA Cancer J Clin 2018;68:394-424.

2. Yang Z, Zeng H, Xia R, et al. Annual cost of illness of stomach and esophageal cancer patients in urban and rural areas in China: A multi-center study. Chin J Cancer Res 2018;30:439-48.

3. Zeng $\mathrm{H}$, Chen $\mathrm{W}$, Zheng $\mathrm{R}$, et al. Changing cancer survival in China during 2003-15: a pooled analysis of 17 population-based cancer registries. Lancet Glob Health 2018;6:e555-67.

4. Reinhorn D, Jacobi O, Icht $\mathrm{O}$, et al. Treatment beyond progression with immune checkpoint inhibitors in nonsmall-cell lung cancer. Immunotherapy 2020;12:235-43.

5. Kojima T, Shah MA, Muro K, et al. Randomized Phase III KEYNOTE-181 Study of Pembrolizumab Versus Chemotherapy in Advanced Esophageal Cancer. J Clin Oncol 2020;38:4138-48.

6. Beaver JA, Hazarika M, Mulkey F, et al. Patients with melanoma treated with an anti-PD-1 antibody beyond RECIST progression: a US Food and Drug Administration pooled analysis. Lancet Oncol 2018;19:229-39.

7. Shah MA, Kojima T, Hochhauser D, et al. Efficacy and Safety of Pembrolizumab for Heavily Pretreated Patients With Advanced, Metastatic Adenocarcinoma or Squamous Cell Carcinoma of the Esophagus: The Phase 2 KEYNOTE-180 Study. JAMA Oncol 2019;5:546-50.

Cite this article as: Peng P, Xiao Y, Zhao Z, Sun C, Wu D, Chen Y, Zhang L. Treatment beyond progression with chemo-immunotherapy in an advanced esophageal squamous cell carcinoma patient: a case report. Transl Cancer Res 2021;10(11):4973-4978. doi: 10.21037/tcr-21-1395
8. Huang J, Xu B, Mo H, et al. Safety, Activity, and Biomarkers of SHR-1210, an Anti-PD-1 Antibody, for Patients with Advanced Esophageal Carcinoma. Clin Cancer Res 2018;24:1296-304.

9. Doi T, Piha-Paul SA, Jalal SI, et al. Safety and Antitumor Activity of the Anti-Programmed Death-1 Antibody Pembrolizumab in Patients With Advanced Esophageal Carcinoma. J Clin Oncol 2018;36:61-7.

10. Sun JM, Shen L, Shah MA, et al. Pembrolizumab plus chemotherapy versus chemotherapy alone for first-line treatment of advanced oesophageal cancer (KEYNOTE-590): a randomised, placebo-controlled, phase 3 study. Lancet 2021;398:759-71.

11. Escudier B, Motzer RJ, Sharma P, et al. Treatment Beyond Progression in Patients with Advanced Renal Cell Carcinoma Treated with Nivolumab in CheckMate 025. Eur Urol 2017;72:368-76.

12. Long GV, Weber JS, Larkin J, et al. Nivolumab for Patients With Advanced Melanoma Treated Beyond Progression: Analysis of 2 Phase 3 Clinical Trials. JAMA Oncol 2017;3:1511-9.

13. Stinchcombe TE, Miksad RA, Gossai A, et al. RealWorld Outcomes for Advanced Non-Small Cell Lung Cancer Patients Treated With a PD-L1 Inhibitor Beyond Progression. Clin Lung Cancer 2020;21:389394.e3.

14. Topalian SL, Taube JM, Anders RA, et al. Mechanismdriven biomarkers to guide immune checkpoint blockade in cancer therapy. Nat Rev Cancer 2016;16:275-87.

15. Schulze AB, Evers G, Görlich D, et al. Tumor infiltrating $\mathrm{T}$ cells influence prognosis in stage I-III non-small cell lung cancer. J Thorac Dis 2020;12:1824-42.

16. Campoli M, Ferrone S. HLA antigen changes in malignant cells: epigenetic mechanisms and biologic significance. Oncogene 2008;27:5869-85.

17. Mehta AM, Jordanova ES, Kenter GG, et al. Association of antigen processing machinery and HLA class I defects with clinicopathological outcome in cervical carcinoma. Cancer Immunol Immunother 2008;57:197-206. 